\title{
Hopf Bifurcation in an Augmented IS-LM Linear Business Cycle Model with Two Time Delays
}

\author{
Firdos Karim \\ Amity Institute of Applied Sciences, \\ Amity University, Noida, Sector-125, 201301, U. P., India. \\ E-mail: meticulous2000@gmail.com \\ Sudipa Chauhan \\ Amity Institute of Applied Sciences, \\ Amity University, Noida, Sector-125, 201301, U. P., India. \\ Corresponding author: sudipachauhan@ gmail.com \\ Sumit Kaur Bhatia \\ Amity Institute of Applied Sciences, \\ Amity University, Noida, Sector-125, 201301, U. P., India. \\ E-mail: sumit2212@gmail.com \\ Joydip Dhar \\ Department of Mathematics, \\ Atal Bihari Vajpayee Indian Institute of Information Technology and Management, \\ Gwalior, M. P., India. \\ E-mail: jdhar@iiitm.ac.in
}

(Received March 4, 2019; Accepted October 22, 2019)

\begin{abstract}
This paper deals with the amalgamated basic IS-LM business cycle model with Kaldor's growth model to form an augmented model. Pertaining to substantial evidence, IS-LM model in paradigm with a specific economic extension (Kaldor-Kalecki Business cycle model in our case) provides an adept explanation of a developing but strong economy like that of our country. Occurring in the equation of capital accumulation, the two time delays are a result of the assumption in the investment function being both income and capital stock dependent in past period and maturity period. Investigating a model combined with capital accumulation is both interesting and important. From economist point of view, production without capital is impossible to even imagine. Moreover capital accumulation is impeccable to largescale production, specialisation and creation of employment opportunities. In our model ' $\mathrm{I}$ ' the investment function, 'S' the savings function and ' $\mathrm{L}$ ' the demand for money are depending linearly on their arguments. We adhere to a linear model, contrary to the popular belief of non- linear models being the undisputed style for modern economics. The model is first shown to be mathematically and economically poised. The local stability of boundary and interior equilibrium points has been investigated. Three cases arise, pertaining to two time delays. System dynamics exhibits mutation under the influence of time delays and may clinch or discharge its local stability when subjected to the latter. Hopf bifurcation occurs when the delay parameter crosses a critical value.
\end{abstract}

Keywords- Business cycle model, Time delay, Capital accumulation, IS-LM model, Hopf bifurcation.

\section{Introduction}

The Indian economy is the world's seventh largest according to market exchange rates and has been a mixed economy post-independence. Business cycles correspond to the protean nature of economic growth and the versatile phases our economy travels through namely expansion, contraction and recovery. Hence, the study of movement of economy through business cycles is 
International Journal of Mathematical, Engineering and Management Sciences

Vol. 5, No. 3, 518-528, 2020

https://doi.org/10.33889/IJMEMS.2020.5.3.043

indispensable. As time delays stand inevitable in economic framework, it also encourages the economist coherently to detach and see the intermingling shackles of events and consequences separately and map the resulting product, oscillating between the intertwingled nature of our monetary activities. With the advent of time delay in investment in 1935 (Frisch and Holme, 1935) there has been ample of literature spanning the extended IS-LM model with time delays (Torre, 1977). Zhou and Li (2009) investigated an IS-LM model with two time delays in capital accumulation equation. In our view, very few papers have emulsified linear models with dual time delays in explaining economic phenomenon, which are nonlinear in nature. The paper is organized as follows. Decent emphasis is laid on the framework of the problem in Section 2. Relevance of IS-LM model in the context of Indian economy and Capital accumulation as a synonym for growth find a fair portion in the proposed discussion. Also, ample amount of space is given to linear business cycle models and objective view on the popular belief of non- linear models being the undisputed style for modern economics. The augmented IS-LM model is introduced in Section 3 which is an amalgamation of basic IS-LM business cycle model with Kaldor Kalecki's model with delay. Model development is discussed through Macroeconomic Flowchart. Beginner's approach is attempted. Section 4 showcases the qualitative behavior of the model along with linear stability analysis. Three cases are discussed, pertaining to two time delays. The last section delivers the insight of the paper and relevant findings. Further research paradigm are outlined.

\section{Economic Milieux}

\subsection{IS-LM Model and the Kaleidoscope of Indian Markets}

The Investment Saving - Liquidity Preference Money Supply model has been the epicentre of macroeconomic modelling since its introduction. Graphically it showcases the intersection of the IS curve (income-expenditure model)involving demand and the LM curve(money available for investing) involving supply. We dedictae a few lines here neutralizing the criticsm surrounding the model. IS-LM model is a summary of the analytical core of Keyne's general theory of employment, interest and money. This model represents a compact portion of the greater theory and therefore the expectation of it dissolving every related macroeconomic process is relatively a little too much. A more cardinal question arising here is how appropriately does the IS-LM model fit into the developing economy of our country. India can be termed as a strong developing economy in lieu of the scio-political arena that stands to be approriately stable. Relevance and relation of a model (the augmented IS-LM model in our case) is of paramount importance given the dynamic factors affecting directly or indirectly to the economic process. Substanial research work (Ahmed, 2005) digs firmly yet within limits to explore the extent to which the dynamic properties of the estimated model of our country match with the concepts of the IS-LM model. The results advocate our stand on the model and its audacity to explain the macroeconomic cycles in the Indian subcontinent.

\subsection{Capital Accumulation: Pillar of Progress}

Investigating a model combined with capital accumulation is both interesting and important. From economist point of view, production without capital is impossible to even imagine. The reasons supporting this statement are surplus in number. We restrict ourselves to the intention of this paper. As capital accumulation enters the room, it brings specialisation and large-scale production with it. Both of them apart from bringing down the cost of production per unit, offer increased output and productivity. Humongous unemployment and under-employment is prominent in both urban and rural areas because the rate at which capital stock moves in fails to match with the rate of growing population. Hence we rest our case of specifically targeting 'Capital Accumulation' and introducing time delays into it. As investigated by Cai (2005) with time lag proposed in capital accumulation, it was propagated that profit's saved part is invested and increment in capital is attributed to 
International Journal of Mathematical, Engineering and Management Sciences

Vol. 5, No. 3, 518-528, 2020

https://doi.org/10.33889/IJMEMS.2020.5.3.043

investment decisions taken in the past. capital. Through the basic model (Zhou and Li, 2009) it is clear that investment occurring in the equation of capital accumulation relies on the aspect of income adhering to the time decisions on investment are conducted and on capital stock corresponding to the phase when investment is finished. Inclusion of dual delay in the equation of capital accumulation propagates our assumption taken in investment function present in the capital accumulation equation being both income and capital stock dependent in past period and maturity period. The delays, respectively, represent the time employed in order that the capital is used for production and the necessary time so that the capital is depreciated.

\subsection{Linear Approach to the Problem}

Asymmetric dimension of business cycles is nearly an undebated style for expressing modern economies. It is a widely accepted notion that nonlinear models are in better position to explain macroeconomic phenomenon and monetary activities. All said and done, trifle evidence exists vouching for substantial performance of nonlinear models over linear ones. In a recent exploration, Lopes and Zsurkis (2019) have used quarterly data (for 29 countries and the euro zone) while adopting a purely testing approach to check the necessity to adhere to nonlinear models to encapsulate business cycle data. Their results advocate the fact of business models not being nonlinear every time as strongly as the common notion that linear models fail noticeably always (Lopes and Zsurkis, 2019). It would be relevant to mention here that the intent is not to prove the supremacy of former over latter or vice-versa but to highlight the importance of linear models in general and supporting the choice of one in our case. The empirical evidence provided by (Lopes and Zsurkis, 2019) raises a question on the slapdash acceptance of nonlinearity of business cycles at global level. While it is omnipresent to some extent, its mere presence is not firm enough to be lauded with global qualifications. The authors suggest that India with seven other countries has very weak evidence of non-linearity at certain frequencies, nevertheless to mention these studies had limitations. In the wake of these facts, we have proceeded with a linear model.

\section{Proposed Mathematical Model}

The IS-LM Model (Zhou and Li, 2009) establishes a general equilibrium between the real sector of goods and services and the monetary sector spanning money market.

$$
\begin{gathered}
\dot{Y}=\alpha(I(Y, r)-S(Y, r)) \\
\dot{r}=\beta(L(Y, r)-\bar{M})
\end{gathered}
$$

and Kaldor's model is given by

$$
\begin{gathered}
\dot{Y}=\alpha(I(Y, K)-S(Y, K)) \\
\dot{K}=I(Y, K)-\delta K
\end{gathered}
$$

This model relies on the assumption of Keynesian full employment and that technical progress is dependent on the rate of capital accumulation. The combination of these two gives us the augmented IS-LM model,

$$
\begin{gathered}
\dot{Y}=\alpha(I(Y, K, r)-S(Y, r)) \\
\dot{r}=\beta(L(Y, r)-\bar{M}) \\
\dot{K}=I(Y, K, r)-\delta K .
\end{gathered}
$$

As per the investigation done by Cai (2005) discussed in the previous section we have, 
International Journal of Mathematical, Engineering and Management Sciences

Vol. 5, No. 3, 518-528, 2020

https://doi.org/10.33889/IJMEMS.2020.5.3.043

$$
\begin{gathered}
\dot{Y}=\alpha(I(Y, r)-S(Y, r)) \\
\dot{r}=\beta(L(Y, r)-\bar{M}) \\
\dot{K}=I(Y(t-T), K, r)-\delta K .
\end{gathered}
$$

Table 1 covers all the variables and parameters that have been used in the model.

Table 1. Variables and Parameters

\begin{tabular}{|c|c|}
\hline Variables and Parameters & Relevance \\
\hline $\mathbf{I}$ & Investment \\
\hline $\mathbf{S}$ & Savings \\
\hline $\mathbf{L}$ & Liquidity preference(demand for money) \\
\hline $\mathbf{K}$ & Capital stock \\
\hline $\mathbf{Y}$ & Gross Product \\
\hline $\mathbf{r}$ & Interest Rate \\
\hline $\mathbf{T}$ & Time delay \\
\hline$\overline{\mathbf{M}}$ & Constant money supply \\
\hline $\boldsymbol{\alpha}$ & Adjustment coefficient in the goods market, $\alpha>0$ \\
\hline $\boldsymbol{\beta}$ & Adjustment coefficient in the money market, $\beta>0$ \\
\hline $\boldsymbol{\delta}$ & Depreciation rate of the capital stock, $0 \leq \delta \leq 1$. \\
\hline
\end{tabular}

We have used macroeconomic flowcharts (Reingewertz, 2013) to explain the concept and steps involved in the model.Our macroeconomic flowchart encapsulates Goods and services market in pink, money market in green and output in voilet. Black arrows span positive correlation whereas red arrows span negavtive correlation. Intermediate equations are marked by blue. Resulting model equations stand with orange background.

3.1 The proposed flowchart illustrated in Figure 1 covers the following assumptions,

(i) As per the LM curve, if government increases the money supply ' $M$ ' the line representing it shifts to the right creating a new money supply $M$ '. In order to achieve new equilibrium in the existing LM curve, the rate of interest decreases in IS-LM Model (n.d.).

(ii) When the liquidity preference curve or demand curve is shifted to the right, in order to achieve equilibrium the interest rate also increases in IS-LM Model (n.d.).

Zak (1999) proposed the following delay ordinary differential equation to describe the dynamics of the solow growth model, $\dot{K}(\boldsymbol{t})=\boldsymbol{s} \boldsymbol{f}(\boldsymbol{K}(\boldsymbol{t}-\boldsymbol{\tau}))-\boldsymbol{\delta} \boldsymbol{K}(\boldsymbol{t}-\boldsymbol{\tau})$.

$\mathrm{K}(\mathrm{t})$ implies the capital at time $\mathrm{t}$, neoclassical production function is given by $\mathrm{f}(\mathrm{K}(\mathrm{t})$ ) and ' $\mathrm{s}$ ' is the constant savings rate (Bianca et al., 2013). As per the cyclic behaviour in the economic system, assumption has been taken that the growth of the amount of capital at time $t$ is a function of the total output of capital at time (Bianca et al., 2013). Generalizing this idea, we have assumed that investment function present in capital accumulation is both income and capital stock dependent in past period and maturity period (IS-LM Model (n.d.)). 
International Journal of Mathematical, Engineering and Management Sciences

Vol. 5, No. 3, 518-528, 2020

https://doi.org/10.33889/IJMEMS.2020.5.3.043

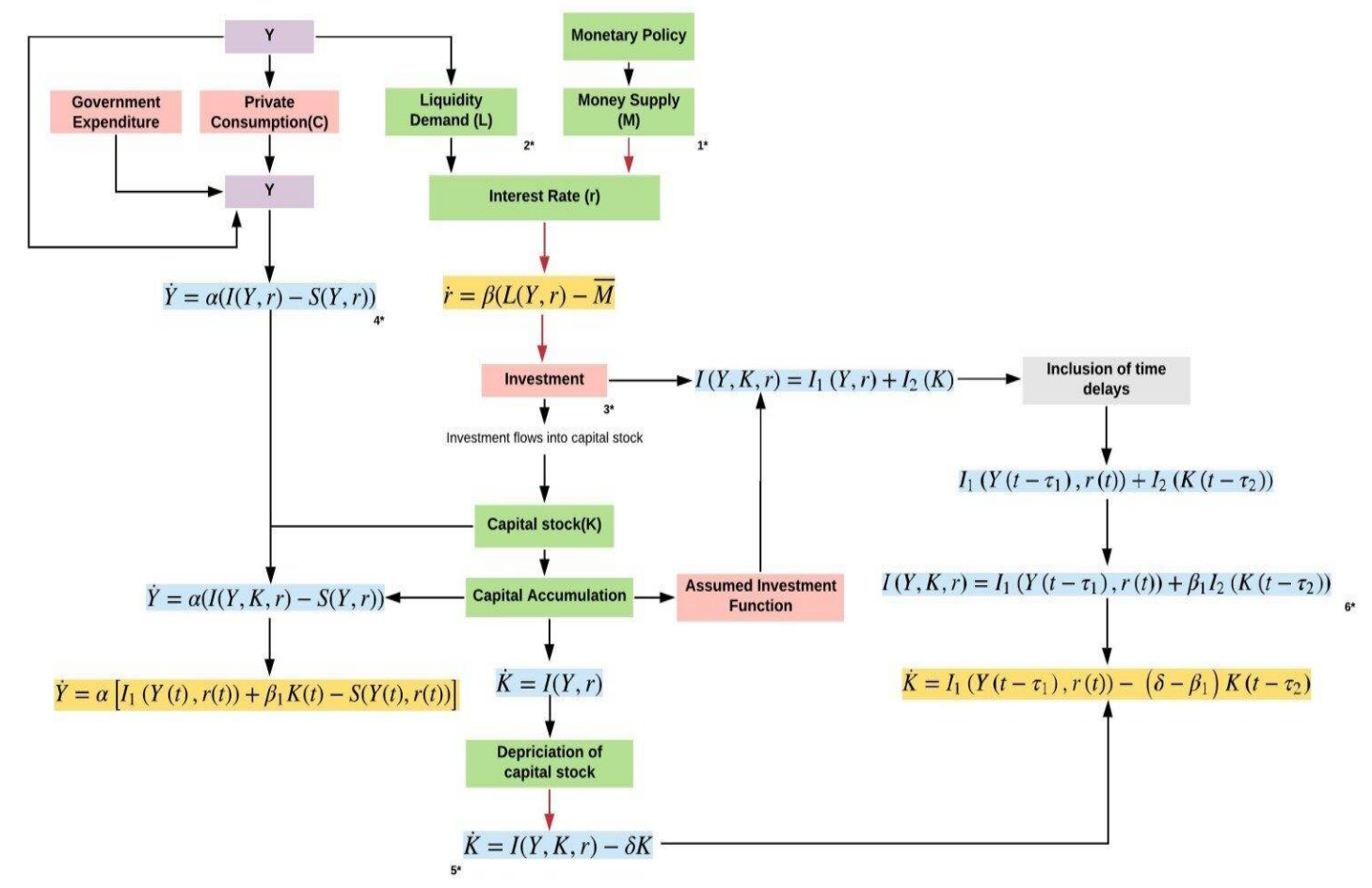

Figure 1. Macroeconomic flowchart

(iii) Gross product is a function of Investment and savings. Here ' $\boldsymbol{\alpha}$ ' is positive and measures the reaction of the system to the difference between investment and saving.

(iv) As capital stock flows in, investment becomes a function of capital accumulation. Stating the depreciation of capital stock, ' $\boldsymbol{\beta}$ ' is negative in nature.

(v) This is an extension of point no. 3 and introduces ' $\boldsymbol{\mu}$ ' as an adjusment coefficient to the previous generalized two time delay equation, where $-\mathbf{1}<\boldsymbol{\mu}<\mathbf{0}$ is propensities to investment $\boldsymbol{I}_{\mathbf{2}}(\boldsymbol{K})$ with respect to capital stock.

Hence, the amalgamated IS-LM model under consideration would be

$$
\begin{aligned}
& \dot{Y}=\alpha\left[I_{1}(Y(t), r(t))+\mu K(t)-S(Y(t), r(t))\right] \\
& \dot{r}(t)=\beta[L(Y(t), r(t)-\bar{M}] \\
& \dot{K}=I_{1}\left(Y\left(t-\tau_{1}\right), r(t)\right)-(\delta-\mu) K\left(t-\tau_{2}\right)
\end{aligned}
$$

\footnotetext{
Macroeconomic flowcharts (Reingewertz, 2013) are constructed using a series of boxes connected with arrows. Economic Variables which belong to the same class share similar colour background. Casual relationships between the variables is depicted by arrows connecting the boxes. Arrows can point in both single as well as two or more directions. Red arrows indicate a negative correlation between the variables and green arrows depict positive correlation. However in our case, we have used black arrows on a common basis for positive correlation. The flow chart is built in a hierarchical manner to promote the order of mechanism. But it does not follow the traditional "one step at a time" exposition. In real world situation there is no natural starting point and the dynamics are circular. Hence an increase in one variable influences other variables.
} 
International Journal of Mathematical, Engineering and Management Sciences

Vol. 5, No. 3, 518-528, 2020

https://doi.org/10.33889/IJMEMS.2020.5.3.043

Another pertinent assumption is the linearity of the functions for investment, savings, and liquidity (demand for money) .

$$
\begin{aligned}
& I=\eta Y-\beta_{1} r \\
& S=l_{1} Y+\beta_{2} r \\
& L=l_{2} Y-\beta_{3} r
\end{aligned}
$$

where, $\eta, \delta_{1}, \beta_{1}, \beta_{2}, \beta_{3}, l_{1}, l_{2}$, are all positive constants.

\section{Analytical Behavior of the Model}

\subsection{Steady State Point of the System}

Equilibria $\left(Y^{*}, r^{*}, K^{*}\right)$ of the proposed system stands at

$$
\begin{gathered}
K^{*}=\frac{1}{\delta-\mu}\left[\eta Y^{*}-\frac{\beta_{1}}{\beta_{2}}\left(l_{2} Y^{*}-\bar{M}\right)\right] \\
r^{*}=\frac{1}{\beta_{3}}\left(l_{2} Y^{*}-\bar{M}\right) \\
Y^{*}=\frac{\left(\beta_{1}+\beta_{2}\right) r^{*}-\mu K^{*}}{\eta-l_{1}} \quad \eta>l_{1}, \quad\left(\beta_{1}+\beta_{2}\right) Y^{*}>\mu K^{*} .
\end{gathered}
$$

\subsection{Local Stability Analysis}

The linearization of the system is done through jacobian and the characteristic equation corresponding to $\left(Y^{*}, r^{*}, K^{*}\right)$ si given by:

$$
U\left(\lambda, \tau_{1}, \tau_{2}\right)=P(\lambda)+D(\lambda) e^{-\lambda \tau_{1}}+E(\lambda) e^{-\lambda \tau_{2}}=0
$$

where

$$
\begin{aligned}
& P(\lambda)=\lambda^{3}+\left[-\alpha\left(\eta-l_{1}\right)+\beta \beta_{3}\right] \lambda^{2}+\left[-\alpha\left(\eta-l_{1}\right) \beta \beta_{3}+\alpha\left(\beta_{1}+\beta_{2}\right) \beta l_{2}\right] \lambda+\alpha \beta \mu \beta_{1} l_{2} \\
& =\lambda^{3}+p_{1} \lambda^{2}+p_{2} \lambda+p_{3} \\
& D(\lambda)=-\eta \alpha \mu \lambda-\eta \alpha \beta \mu \beta_{3}=d_{1} \lambda+d_{2} \\
& E(\lambda)=(\delta-\mu) \lambda^{2}+\left[-\alpha\left(\eta-l_{1}\right)(\delta-\mu)+\beta \beta_{3}(\delta-\mu)\right] \lambda-\alpha\left(\eta-l_{1}\right) \beta \beta_{3}(\delta-\mu) \\
& +\alpha\left(\beta_{1}+\beta_{2}\right) \beta l_{2}(\delta-\mu)=e_{1} \lambda^{2}+e_{2} \lambda+e_{3} \text {. }
\end{aligned}
$$

We now consider three cases pertaining to time delays.

Case 1: $\tau_{1}=0, \tau_{2}=0$

The characteristic polynomial Eq.(2) reduces to

$$
U(\lambda, 0,0)=P(\lambda)+D(\lambda)+E(\lambda)=\lambda^{3}+\left(p_{1}+e_{1}\right) \lambda^{2}+\left(p_{2}+d_{1}+e_{2}\right) \lambda+\left(p_{3}+d_{2}+e_{3}\right)=0
$$

In accordance with Routh-Hurwitz criterion, equilibrium point stands to be stable if

$$
\left(\mathrm{X}_{1}\right) p_{2}+e_{1}>0, \quad\left(p_{1}+e_{1}\right)\left(p_{2}+d_{1}+e_{2}\right)\left(p_{3}+d_{2}+e_{3}\right)>0
$$

Case 2: $\tau_{1}=0, \tau_{2} \neq 0$

Putting $\tau_{1}=0$ in (2), the characteristic equation becomes

$$
\begin{aligned}
U\left(\lambda, 0, \tau_{2}\right) & =P(\lambda)+D(\lambda)+E(\lambda) e^{-\lambda \tau_{2}} \\
& =\lambda^{3}+p_{1} \lambda^{2}+\left(p_{2}+d_{1}\right) \lambda+\left(p_{3}+d_{2}\right)+e^{-\lambda \tau_{2}}\left(e_{1} \lambda^{2}+e_{2} \lambda+e_{3}\right)=0
\end{aligned}
$$


International Journal of Mathematical, Engineering and Management Sciences

Vol. 5, No. 3, 518-528, 2020

https://doi.org/10.33889/IJMEMS.2020.5.3.043

Pertaining to the methodology in Beretta and Kuang (2002) the imaginary solutions of Eq.(4) should be found.Let $\lambda=i \omega$ be these solutions. We know that $U(O, O, O)=p_{3}+d_{2} \neq 0$.

Following assumption $\left(X_{1}\right)$ (It implies that imaginary axis cannot be crossed by real values). We further assume that $\omega>0$. Finding roots $\lambda=i \omega$ of (4), because $\lambda=-i \omega$ is also a root of (4). Putting these values in (4) and evaluating the real and imaginary parts, it follows that

$\sin \left(\omega \tau_{2}\right)=\operatorname{Im}\left(\frac{P(\omega i)+D(\omega i)}{E(\omega i)}\right), \cos \left(\omega \tau_{2}\right)=-\operatorname{Re}\left(\frac{P(\omega i)+D(\omega i)}{E(\omega i)}\right)$

A necessary condition to have $\omega$ as a solution of (4) is that $\omega$ must be a root of the following equation:

$$
\begin{aligned}
F(\omega)= & |P(\omega i)+D(\omega i)|^{2}-|E(\omega i)|^{2} \\
= & \omega^{6}+\left[p_{1}^{2}-2\left(p_{2}+d_{1}\right)-e_{1}^{2}\right] \omega^{4}+\left[\left(p_{2}+d_{1}\right)^{2}-2 p_{1}\left(p_{3}+d_{2}\right)+2 e_{3} e_{1}-e_{2}^{2}\right] \omega^{2}+ \\
& {\left[\left(p_{3}+d_{2}\right)^{2}-e_{3}^{2}\right] } \\
= & 0
\end{aligned}
$$

Let $x=\omega^{2}$.Then

$F(x)=x^{3}+A x^{2}+B x+C$

where,

$A=p_{1}^{2}-2\left(p_{2}+d_{1}\right)-e_{1}^{2}, \quad B=\left(p_{2}+d_{1}\right)^{2}-2 p_{1}\left(p_{3}+d_{2}\right)+2 e_{3} e_{1}-e_{2}^{2}$,

$C=\left(p_{3}+d_{2}\right)^{2}-e_{3}^{2}$.

Following the conclusion by De Cesare and Sportelli (2005) we assume that

$\left(X_{2}\right)$ anyone of $A \geq 0, C<0 ; B \leq 0, C<0 ; A<0, B>0, C<0, \triangle>0 ; A<0$,

$B=0, C=0 ; B<0, C=0$;

$\left(X_{3}\right)$ anyone of $A<0, B>0, C>0, \triangle<0 ; A<0, B>0, C=0, A^{2}>4 B$

$\left(X_{4}\right)$ anyone of $A<0, B>0, C<0, \triangle<0$;

$\left(X_{5}\right)$ anyone of $A \geq 0, B \geq 0, C \geq 0 ; C>0, \triangle>0$

where the discriminant is given by $\triangle=\frac{F(K)^{2}}{4}+\frac{F^{\prime}(K)^{3}}{9}, k=\frac{-4}{3}$ (In accordance with the reduced form Eq.7). Hence we can deduce that ,

Lemma 4.3 We can establish the following results for Eq.(7)

a. There exist only one positive real root $\omega_{1}$ for $\mathbf{E q} .(7)$, if $\left(X_{2}\right)$ holds;

b. There exist two distinct positive real root $\omega_{2}, \omega_{3}\left(\right.$ setting $\left.\omega_{2}, \omega_{3}\right)$ for Eq. (7), if $\left(X_{3}\right)$ holds;

c. There exist three distinct positive real roots $\boldsymbol{\omega}_{1}<\boldsymbol{\omega}_{2}<\boldsymbol{\omega}_{3}$ for $\boldsymbol{E q}$. (7), if $\left(\boldsymbol{X}_{4}\right)$ holds;

d. There is no positive real root for $\boldsymbol{E} \boldsymbol{q} .(\mathbf{7})$, if $\left(\boldsymbol{X}_{\mathbf{5}}\right)$ holds.

It is relevant to mention here that if and only if $\tau_{2}^{*}=\frac{\phi\left(\omega^{*}\right)+2 n \pi}{\omega^{*}} n \in N, \phi \epsilon[0,2 \pi]$, then a solution $\omega$ of Eq. (7) is also a solution of the characteristic equation Eq.(5).

Recalling the Lemma in Cooke and Grossman (1982), we get that 
International Journal of Mathematical, Engineering and Management Sciences

Vol. 5, No. 3, 518-528, 2020

https://doi.org/10.33889/IJMEMS.2020.5.3.043

Lemma Let $f(\lambda, \tau)=\lambda^{2}+a \lambda+b \lambda e^{-\lambda \tau}+c+d e^{-\lambda \tau}$ where a,b,c,d, $\tau$ are real numbers and $\tau \geq 0$. Then, as $\tau$ varies, the sum of the multiplicities of zeroes of " $\mathrm{f}$ " in the open righthalf-plane can change only if a zero appears on or crosses the imaginary axis. Using this lemma, we put forward,

Lemma 4.4 Considering $\mathrm{Eq}(4)$ we have

a. There exist only one pair of roots that are imaginary $\pm \iota \omega_{1}$ for Eq. (4), if $\left(\mathrm{H}_{2}\right)$ holds and $\tau_{2}=\tau_{2 . n}^{1}$.

b. There exist two pairs of purely imaginary roots $\pm \iota \omega_{2}, \pm \iota \omega_{3}$ for Eq.(4), if $\left(\mathrm{H}_{3}\right)$ holds and $\tau_{2}=\tau_{2 . n}^{2}, \tau_{2 . n}^{3}$.

c. There exist three pairs of purely imaginary roots $\pm \iota \omega_{1}, \pm \iota \omega_{2}, \pm \iota \omega_{3}$ for Eq.(4), if $\left(H_{4}\right)$ holds and $\tau_{2}=\tau_{2 . n}^{1}, \tau_{2 . n}^{2}, \tau_{2 . n}^{3}$.

d. There exist only no imaginary roots for $\mathbf{E q} .(4)$, if $\left(\boldsymbol{H}_{5}\right)$ holds where

$$
\begin{aligned}
& \tau_{2 . n}^{1}=\frac{1}{\omega_{1}} \cos ^{-1}\left\{\frac{\left(p_{1} e_{1}-e_{2}\right) \omega_{1}^{4}+\left[\left(p_{2}+d_{1}\right) e_{2}-p_{1} e_{3}-\left(p_{3}+d_{2}\right) e_{1}\right] \omega_{1}^{2}+\left(p_{3}+d_{2}\right) e_{3}}{\left(e_{1} \omega_{1}^{2}-e_{3}\right)^{2}+\left(e_{2} \omega_{1}\right)^{2}}\right\} \\
& +\frac{2 n \pi}{\omega_{1}} \\
& \tau_{2 . n}^{2}=\frac{1}{\omega_{2}} \cos ^{-1}\left\{\frac{\left(p_{1} e_{1}-e_{2}\right) \omega_{2}^{4}+\left[\left(p_{2}+d_{1}\right) e_{2}-p_{1} e_{3}-\left(p_{3}+d_{2}\right) e_{1}\right] \omega_{2}^{2}+\left(p_{3}+d_{2}\right) e_{3}}{\left(e_{1} \omega_{2}^{2}-e_{3}\right)^{2}+\left(e_{2} \omega_{2}\right)^{2}}\right\} \\
& +\frac{2 n \pi}{\omega_{2}} \\
& \tau_{2 . n}^{3}=\frac{1}{\omega_{3}} \cos ^{-1}\left\{\frac{\left(p_{1} e_{1}-e_{2}\right) \omega_{3}^{4}+\left[\left(p_{2}+d_{1}\right) e_{2}-p_{1} e_{3}-\left(p_{3}+d_{2}\right) e_{1}\right] \omega_{3}^{2}+\left(p_{3}+d_{2}\right) e_{3}}{\left(e_{1} \omega_{3}^{2}-e_{3}\right)^{2}+\left(e_{2} \omega_{3}\right)^{2}}\right\} \\
& +\frac{2 n \pi}{\omega_{3}} \text {. }
\end{aligned}
$$

Lemma 4.5 Suppose $\tau_{2}^{*}$ depicts an entity of either sequence $\left\{\tau_{2 . n}^{1}\right\}$ or $\left\{\tau_{2 . n}^{2}\right\}$ or $\left\{\tau_{2 . n}^{3}\right\}$, then the given transvesality condition are fulfilled:

$$
\begin{aligned}
& \operatorname{sign}\left\{\left.\frac{d R e(\lambda)}{d \tau_{2}}\right|_{\tau_{2}=\tau_{2}^{*}}\right\}=\operatorname{sign} F^{\prime}\left(x^{2}\right)=\operatorname{sign}\left(3 x^{3}+2 A x^{2}+B x\right) . \\
& \operatorname{sign}\left\{\left.\frac{d \operatorname{Re}(\lambda)}{d \tau_{2}}\right|_{\tau_{2}=\tau_{2 . n}^{1}}\right\}>0, \operatorname{sign}\left\{\left.\frac{d \operatorname{Re}(\lambda)}{d \tau_{2}}\right|_{\tau_{2}=\tau_{2 . n}^{2}}\right\}<0, \operatorname{sign}\left\{\left.\frac{d \operatorname{Re}(\lambda)}{d \tau_{2}}\right|_{\tau_{2}=\tau_{2 . n}^{3}}\right\}>0
\end{aligned}
$$

Proof. For transversality conditions we have from Eq.(2)

$\operatorname{Re}\left[\frac{d \lambda}{d \tau_{2}}\right]^{-1}=\frac{\left(3 x^{3}+2 A x^{2}+B x\right)}{\wedge}=\frac{\mathrm{x}\left(3 x^{2}+2 A \mathrm{x}+B\right)}{\wedge}=\frac{\mathrm{xF}^{\prime}(\mathrm{x})}{\wedge}\left(\right.$ where $\left.\mathrm{F}(\mathrm{x})=x^{3}+A x^{2}+B x+C\right)$

Here $\Lambda=\left(e_{2} \omega^{2}\right)^{2}+\left(e_{3} \omega-e_{1} \omega^{3}\right)^{2}>0$. Hence, $\operatorname{sign}\left\{\frac{x \mathrm{~F}^{\prime}(\mathrm{x})}{\wedge}\right\} \neq 0$.

Lemma 4.6 We consider the following for Eq. (4)

a. Considering if $\left(\boldsymbol{X}_{\mathbf{1}}\right)$ and $\left(\boldsymbol{X}_{\mathbf{2}}\right)$ hold, then all roots of Eq.(4) have negative real parts when $\boldsymbol{\tau}_{\mathbf{2}} \boldsymbol{\epsilon}\left[\mathbf{0}, \boldsymbol{\tau}_{\mathbf{2} . \mathbf{0}}^{\mathbf{1}}\right)$ and Eq. (4) has at least one root with positive real part when $\boldsymbol{\tau}_{\mathbf{2}}>\boldsymbol{\tau}_{\mathbf{2 . 0}}^{\mathbf{1}}$.

$b$. Considering if $\left(\boldsymbol{X}_{\mathbf{1}}\right)$ and $\left(\boldsymbol{X}_{\mathbf{3}}\right)$ hold, then there exist $k$ switches from stability to instability 
International Journal of Mathematical, Engineering and Management Sciences

Vol. 5, No. 3, 518-528, 2020

https://doi.org/10.33889/IJMEMS.2020.5.3.043

when the parameters such that $\tau_{2.0}^{3}<\tau_{2.0}^{2}<\tau_{2.1}^{3}<, \ldots,<\tau_{2 . k-2}^{3}<\tau_{2 . k-2}^{2}<\tau_{2 . k-1}^{3}<$ $\boldsymbol{\tau}_{2 . k}^{3}<\boldsymbol{\tau}_{2 . k-1}^{2}$, all roots of Eq.(4) have negative real parts when $\boldsymbol{\tau}_{\mathbf{2}} \boldsymbol{\epsilon}\left(\boldsymbol{\tau}_{\mathbf{2} . n}^{2}, \boldsymbol{\tau}_{\mathbf{2 . n + 1}}^{3}\right), \boldsymbol{\tau}_{\mathbf{2 . - 1}}^{2}=0$, $n=-1,0, \ldots \ldots ., k-1$. When $\boldsymbol{\tau}_{\mathbf{2}} \in\left[\boldsymbol{\tau}_{\mathbf{2 . n}}^{3}, \boldsymbol{\tau}_{\mathbf{2 . n}}^{\mathbf{2}}\right), \boldsymbol{n}=\mathbf{0}, \mathbf{1} \ldots \ldots . \boldsymbol{k}-\mathbf{1}$ and $\boldsymbol{\tau}_{\mathbf{2}}>\boldsymbol{\tau}_{\mathbf{2 . k}}^{\mathbf{3}}$ Eq(4)has at least one root with positive real parts.

c. Considering if $\left(\boldsymbol{X}_{\mathbf{1}}\right)$ not holds but $\left(\boldsymbol{X}_{\mathbf{3}}\right)$ holds, when the parameters such that $\boldsymbol{\tau}_{\mathbf{2 . 0}}^{2}<\boldsymbol{\tau}_{\mathbf{2} . \mathbf{0}}^{\mathbf{3}}<$ $\boldsymbol{\tau}_{\mathbf{2 . 1}}^{2}<, \ldots,<\boldsymbol{\tau}_{\mathbf{2 . k - 1}}^{2}<\boldsymbol{\tau}_{\mathbf{2 . k - 1}}^{3}<\boldsymbol{\tau}_{\mathbf{2 . k}}^{3}<\boldsymbol{\tau}_{\mathbf{2} . \mathbf{k}}^{2}$, there may exist $k$ switches from instability to stability, that is when $\boldsymbol{\tau}_{\mathbf{2}} \boldsymbol{\epsilon}\left[\boldsymbol{\tau}_{\mathbf{2 . n}}^{\mathbf{3}}, \boldsymbol{\tau}_{\mathbf{2 . n + 1}}^{\mathbf{2}}\right)$, and $\boldsymbol{\tau}_{\mathbf{2}}>\boldsymbol{\tau}_{\mathbf{2 . k - 1}}^{\mathbf{3}}, \boldsymbol{\tau}_{\mathbf{2} . \mathbf{1}}^{\mathbf{3}}=0, n=-1,0, \ldots \ldots ., k-2$. Eq.(4) has at least one root with positive real parts. When $\boldsymbol{\tau}_{\mathbf{2}} \boldsymbol{\epsilon}\left[\boldsymbol{\tau}_{\mathbf{2} . n}^{\mathbf{2}}, \boldsymbol{\tau}_{\mathbf{2} . \mathbf{n}}^{3}\right), n=0,1, \ldots \ldots . . . k-1$, all roots of Eq.(4) have negative real parts.

d. There exists at least one stability switch, if $\left(\boldsymbol{X}_{\mathbf{1}}\right)$ and $\left(\boldsymbol{X}_{\mathbf{4}}\right)$ hold.

\section{Proof}

a. Considering ( $\left.\mathbf{X}_{\mathbf{1}}\right)$ and $\left(\mathbf{X}_{\mathbf{2}}\right)$ hold, then the equilibrium of the Eq.(4)is stable and the Eq.(4) has complex root with negative real parts for $\boldsymbol{\tau}_{\mathbf{2}}=\mathbf{0}$, and also for $\boldsymbol{\tau}_{\mathbf{2}}=\boldsymbol{\tau}_{\mathbf{2 . 0}}^{\mathbf{1}}$ Eq. (4) has purely imaginary roots and the real parts of the root changes continuously increasing with increasing $\tau_{2}$ because of $\operatorname{sign}\left\{\left.\frac{\operatorname{dRe}(\lambda)}{d \tau_{2}}\right|_{\tau_{2}=\tau_{2 . n}^{1}}\right\}>\mathbf{0}$, then for $\tau_{2} \in\left[0, \tau_{2.0}^{1}\right)$ all the roots of Eq. (4) have negative real parts and Eq.(4) has atleast one root with positive real parts when $\boldsymbol{\tau}_{\mathbf{2}}>\boldsymbol{\tau}_{\mathbf{2 . 0}}^{1}$.

b. Because $\boldsymbol{\omega}_{3}>\boldsymbol{\omega}_{2}$ and $\boldsymbol{\tau}_{\mathbf{2}, \mathbf{n}}^{\mathrm{i}}=\frac{\boldsymbol{\phi}\left(\boldsymbol{\omega}_{\mathrm{i}}\right)+\mathbf{2 n \pi}}{\boldsymbol{\omega}_{\mathrm{i}}}, \mathrm{i}=2,3$ are the two corresponding sequences for the time delay $\boldsymbol{\tau}_{\mathbf{2}}$, then $\exists \mathbf{k}, \boldsymbol{\tau}_{\mathbf{2 . k}}^{3}-\boldsymbol{\tau}_{\mathbf{2 . k}-\mathbf{1}}^{3}=\frac{2 \pi}{\omega_{3}}<\frac{2 \pi}{\omega_{2}}=\tau_{\mathbf{2 . k}-\mathbf{1}}^{2}-\tau_{\mathbf{2 . k}-2}^{2}$, and also $\left(\mathbf{X}_{1}\right)$ and $\left(\mathbf{X}_{\mathbf{2}}\right.$ ) hold, then the equilibrium is stable for $\boldsymbol{\tau}_{\mathbf{2}}=\mathbf{0}$. Therefore necessarily $\boldsymbol{\tau}_{\mathbf{2 . 0}}^{3}<\boldsymbol{\tau}_{\mathbf{2 . 0}}^{2}$ (or the multiplicities of roots with negative real parts could become two for Eq.(8) when $\boldsymbol{\tau}_{\mathbf{2}}$ is decreased,it's impossible (Cooke and Grossman,1982), when the parameters are such that $\boldsymbol{\tau}_{2.0}^{3}<\boldsymbol{\tau}_{2.0}^{2}<\tau_{2.1}^{3}<, \ldots,<\tau_{2 . k-2}^{2}<\tau_{2 . k-1}^{3}<\tau_{2 . k}^{3}<\tau_{2 . k-1}^{2}$, we know that there exist a lot of stability switches and the stability of the equilibrium of change a finite of times at most, eventually it becomes unstable.

c. Exactly similar proof exists like that of $b$.

d. If ( $\left.\mathbf{X}_{\mathbf{1}}\right)$ and ( $\left.\mathbf{X}_{\mathbf{4}}\right)$ hold, then the equilibrium of the Eq.(4) is stable and Eq. (4) has three pair of purely imaginary roots $\boldsymbol{\omega}_{1}, \boldsymbol{\omega}_{2}, \boldsymbol{\omega}_{3}$, and Eq.(8) holds, so there exist at least one stability switch at $\boldsymbol{\tau}_{2.0}^{1}$ or $\tau_{2.0}^{3}$.

Emphasising on the theorem in Hale and Jake (1977) and considering the dynamic ananlysis above, we roll down to the following results:

Theorem 4.1 Consider the conditions ( $a, b, c)$ occurring in Lemma 4.6, when they are satisfied then,

a. When $\boldsymbol{\tau}_{\mathbf{2}} \in\left[\mathbf{0}, \boldsymbol{\tau}_{\mathbf{2 . 0}}^{\mathbf{1}}\right)$ the equilibrium $\left(\boldsymbol{Y}^{*}, \boldsymbol{K}^{*}\right)$ is locally asymptotically stable and a Hopf bifurcation occurs when $\tau_{2}=\tau_{2.0}^{1}$ at $\left(\boldsymbol{Y}^{*}, K^{*}\right)$.

b. When $\boldsymbol{\tau}_{\mathbf{2}} \boldsymbol{\epsilon}\{\mathbf{0}\} \cup\left[\boldsymbol{\tau}_{2 . n}^{2}, \boldsymbol{\tau}_{2 . n+1}^{3}\right), \boldsymbol{\tau}_{2 .-1}^{2}=\mathbf{0}, \boldsymbol{n}=-\mathbf{1}, \mathbf{0} \ldots \ldots . \boldsymbol{k}-\mathbf{2}$ the equilibrium $\left(\boldsymbol{Y}^{*}, \boldsymbol{K}^{*}\right)$ is locally asymptotically stable and when $\boldsymbol{\tau}_{\mathbf{2}}=\boldsymbol{\tau}_{\mathbf{2 . n}}^{\boldsymbol{m}} \cup \boldsymbol{\tau}_{\mathbf{2 . k - 1}}^{3}, \mathrm{~m}=2,3, \mathrm{n}=0,1 \ldots \ldots .$. $\mathrm{k}-2$ a Hopf bifurcation occurs.

c. When $\boldsymbol{\tau}_{\mathbf{2}} \in\left[\boldsymbol{\tau}_{\mathbf{2 . n}}^{2}, \boldsymbol{\tau}_{\mathbf{2 . n}}^{3}\right), \mathrm{n}=0,1 \ldots . . \mathrm{k}-1$ the equilibrium $\left(\boldsymbol{Y}^{*}, \boldsymbol{K}^{*}\right)$ is locally asymptotically stable, when $\tau_{2}=\tau_{2 . n}^{m}, \boldsymbol{m}=\mathbf{2}, \mathbf{3}, \boldsymbol{n}=\mathbf{0}, \mathbf{1} \ldots \ldots, \boldsymbol{k}-\mathbf{1}$ a hopf bifurcation occurs. 
International Journal of Mathematical, Engineering and Management Sciences

Vol. 5, No. 3, 518-528, 2020

https://doi.org/10.33889/IJMEMS.2020.5.3.043

Case 3: $\tau_{1} \neq 0, \tau_{2} \neq 0$

Now regarding $\tau_{1}$ as a parameter, we consider Eq.(2) with $\tau_{1}>0$ and $\tau_{2}$ in stable regions. Collecting from Ruan and Wei (2003), we have,

Lemma 4.7 For $\tau_{2}>0$, if all roots of Eq. (4) have negative real parts then there exists a $\tau_{1}{ }^{*}\left(\tau_{2}\right)>$ 0 such that all roots of Eq.( 2) have negative real parts when $0 \leq \tau_{1}<\tau_{1}{ }^{*}\left(\tau_{2}\right)$.

Proof. The left hand side of Eq. (2) is analytic in $\lambda$ and $\tau_{1}$, hence we recollect the Theorem 2.1 of Ruan and Wei (2003) and put up that the sum of the multiplicities of zeros of the left hand side of Eq. (2) in the open right half-plane can change only if a zero on or cross the imaginary axis, as $\tau_{1}$ varies.

Theorem 4.2 Let us consider $\left(\mathrm{X}_{1}\right)$ holds true.

a. For any $\boldsymbol{\tau}_{\mathbf{2}} \in\left[\mathbf{0}, \boldsymbol{\tau}_{\mathbf{2} . \mathbf{0}}^{\mathbf{1}}\right)$, there exists a $\boldsymbol{\tau}_{\mathbf{1}}{ }^{*}\left(\boldsymbol{\tau}_{\mathbf{2}}\right)$, such that the equilibrium of system (1) is locally asymptotically stable when $\boldsymbol{\tau}_{\mathbf{1}} \in\left[\mathbf{0}, \boldsymbol{\tau}_{\mathbf{1}}{ }^{*}\left(\boldsymbol{\tau}_{\mathbf{2}}\right)\right)$, provided $\left(\mathbf{X}_{\mathbf{2}}\right)$ holds.

b. For any $\boldsymbol{\tau}_{\mathbf{2}} \boldsymbol{\epsilon}\left[\boldsymbol{\tau}_{\mathbf{2} . \mathbf{n}}^{\mathbf{2}}, \boldsymbol{\tau}_{\mathbf{2} . \mathbf{n}+\mathbf{1}}^{\mathbf{3}}\right)$, there exists a $\boldsymbol{\tau}_{\mathbf{1}}{ }^{*}\left(\boldsymbol{\tau}_{\mathbf{2}}\right)$, such that the equilibrium of system (1) is locally asymptotically stable when $\boldsymbol{\tau}_{\mathbf{1}} \in\left[\mathbf{0}, \boldsymbol{\tau}_{\mathbf{1}}{ }^{*}\left(\boldsymbol{\tau}_{\mathbf{2}}\right)\right)$, provided $\left(\mathbf{X}_{\mathbf{3}}\right)$ holds.

c. For any $\boldsymbol{\tau}_{\mathbf{2}} \geq \mathbf{0}$, , there exists a $\boldsymbol{\tau}_{\mathbf{1}}{ }^{*}\left(\boldsymbol{\tau}_{\mathbf{2}}\right)$, such that the equilibrium of system (1) is locally asymptotically stable when $\boldsymbol{\tau}_{\mathbf{1}} \in\left[\mathbf{0}, \boldsymbol{\tau}_{\mathbf{1}}{ }^{*}\left(\boldsymbol{\tau}_{\mathbf{2}}\right)\right)$, provided $\left(\mathbf{X}_{\mathbf{5}}\right)$ holds.

Proof. (a and b). We can establish the desired results pertaining to points (a) and (b) of Lemmas 4.6 and 4.7 .

c. Considering Lemma 4.7 there exists a $\tau_{1}{ }^{*}\left(\tau_{2}\right)>0$, such that for $0 \leq \tau_{1}<\tau_{1}{ }^{*}\left(\tau_{2}\right)$ all roots of Eq. (2) have negative real parts. For any $\tau_{2} \geq 0$ all roots of Eq. (4) have negative real parts provided $\left(X_{1}\right)$ and $\left(X_{5}\right)$ hold.

Undoubtedly Hopf bifurcation occurs at $\tau_{1}{ }^{*}\left(\tau_{2}\right)$ provided the conditions of Lemma 4.7 or Theorem 4.2 are met. Surplus stability switches may exist. There may exist no $\tau_{1}{ }^{*}\left(\tau_{2}\right)$ if we let $\tau_{2}$ in unstable region such that when the system (1) is stable in $\tau_{1}{ }^{*}\left(\tau_{2}\right)<\tau_{1}$ it's unstable in $0 \leq$ $\tau_{1}<\tau_{1}^{*}\left(\tau_{2}\right)$.

\section{Conclusion}

Our findings imply that while a single time delay instigates the system to change its qualitative nature, two lags contribute to a change in the behaviour of the system. Fiscal policy lags or time delays are inherent in the context of economy and will continue to interfere with its dynamic nature. There is a specific time after which the capital is available for production, hence policymakers should ensure that the capital stock should be made available within a certain time period so that it can ensure increased output. Failing to which, we will not be able to achieve desired income even when capital is available. As a lead to this research work, impact of three or more-time delays to similar augmented models can be taken into consideration.

\section{Conflict of Interest}

The authors confirm that there is no conflict of interest to declare for this publication. 
International Journal of Mathematical, Engineering and Management Sciences

Vol. 5, No. 3, 518-528, 2020

https://doi.org/10.33889/IJMEMS.2020.5.3.043

\section{Acknowledgments}

The authors would like to appreciate the effort from editors and reviewers. The following research did not claim any particular or general grant from any of the funding agencies i.e., public, commercial or not-for-profit sectors. This paper has been presented in the National Conference ROASET-19 at Ajay Kumar Garg Engineering College, Ghaziabad UP (India) held on 24-25 April 2019.

\section{References}

Ahmed, M. (2005). How well does the IS-LM model fit in a developing economy: the case of India. International Journal of Applied Economics, 2(1), 90-106.

Beretta, E., \& Kuang, Y. (2002). Geometric stability switch criteria in delay differential systems with delay dependent parameters. SIAM Journal on Mathematical Analysis, 33(5), 1144-1165.

Bianca, C., Ferrara, M., \& Guerrini, L. (2013). The time delays' effects on the qualitative behavior of an economic growth model. Abstract and Applied Analysis, 2013, 1-10. http://dx.doi.org/10.1155/2013/901014.

Cai, J. (2005). Hopf bifurcation in the IS-LM business cycle model with time delay. Electronic Journal of Differential Equations, 2005(15), 1-6.

Cooke, K.L., \& Grossman, Z. (1982). Discrete delay, distributed delay and stability switches. Journal of Mathematical Analysis and Applications, 86(2), 592-627.

De Cesare, L., \& Sportelli, M. (2005). A dynamic IS-LM model with delayed taxation revenues. Chaos, Solitons \& Fractals, 25(1), 233-244.

Frisch, R., \& Holme, H. (1935). The characteristic solutions of a mixed difference and differential equation occuring in economic dynamics. Econometrica: Journal of the Econometric Society, 3(2), 225-239.

Hale, \& Jake, K. (1977). Theory of functional differential equations. Springer-Verlag, New York.

IS-LM Model. (n.d.). Retrieved from https://macroeconomicanalysis.com/macroeconomics-wikipedia/is-lmmodel/ , Accessed in February 2019.

Lopes, A.S., \& Zsurkis, G.F. (2019). Are linear models really unuseful to describe business cycle data? Applied Economics, 51(22), 2355-2376.

Reingewertz, Y. (2013). Teaching macroeconomics through flowcharts. International Review of Economics Education, 14, 86-93.

Ruan, S., \& Wei, J. (2003). On the zeros of transcendental functions with applications to stability of delay differential equations with two delays. Dynamics of Continuous, Discrete and Impulsive Systems Series A: Mathematical Analysis, 10(6), 863-874.

Torre, V. (1977). Existence of limit cycles and control in complete keynesian system by theory of bifurcations. Econometrica: Journal of the Econometric Society, 45(6), 1457-1466.

Zak, P.J. (1999). Kaleckian lags in general equilibrium. Review of Political Economy, 11(3), 321-330.

Zhou, L., \& Li, Y. (2009). A dynamic IS-LM business cycle model with two time delays in capital accumulation equation. Journal of Computational and Applied Mathematics, 228(1), 182-187. 